\title{
Platelet-Rich Plasma in Temporomandibular Joint Osteoarthritis Therapy: A 3-Month Follow-Up Pilot Study
}

\author{
Vladimir Machoň ${ }^{1 *}$, Monika Řehořová1, Jiří Šedý1,2 and René Foltán ${ }^{1}$
}

${ }^{1}$ Department of Oral Maxillofacial Surgery, General University Hospital, Prague, Czech Republic

${ }^{2}$ Institute of Physiology, Academy of Sciences of the Czech Republic, Prague, Czech Republic

\begin{abstract}
The use of Platelet-Rich-Plasma (PRP) may provide a new and improved treatment option for early Temporomandibular Joint (TMJ) Osteoarthritis (OA). However, there are no studies on its use in TMJ arthritis in the literature. The present study compares 30 early onsets OA patients divided equally into 3 groups who had experienced no pain reduction following conservative and minimally invasive arthrocentesis and arthroscopy treatment. All patients had retrodiscitis and synovitis on arthroscopic examination and were without systemic joint disease, septic, or autoimmune arthritis. Only patients with unilateral TMJ symptoms were included in this study. Pain intensity was recorded for each patient using a 0-10 VAS scale. Maximum Interincisal Opening (MIO) was also recorded. This assessment was performed at the pretreatment and then at an examination 3 months after administration of 2 intra-articular applications of autologous PRP (Group A), 2 intra-articular injections of Hyaluronic Acid (HA) (Group B), or commencement of conservative therapy only with soft diet and analgetics (Group C). The results after 3 months revealed that intra-articular injection of autologous PRP appeared to be an effective treatment method for patients with early OA in this study. At the 3-month follow-up, patients from group A improved their mouth opening significantly. Majority of the PRP patients (Group A) showed decreased pain. The average pain score before PRP administration was 7, while 3 months after PRP administration the pain score was 3.5. In conclusion, the use of PRP is an effective treatment option for early TMJ OA.
\end{abstract}

Keywords: Platelet-rich plasma; Temporomandibular joint; Osteoarthritis

\section{Introduction}

Osteoarthritis $(\mathrm{OA})$ is characterized by the gradual degeneration of cartilage and subchondral bone due to mechanical strain on joints (micro- and macrotrauma), intraarticular joint disorders (discopathy), or arthritic disease. The effects of free radicals and cytokines (interleukin-1b and tumor necrosis factor alpha) activate protease receptors and subsequently lead to cartilage fibrillation and erosion, osteophyte formation and sclerotization of subchondral bone. Advanced degeneration manifests through flattening of the condyle, joint protuberances, erosion, and disc perforation [1,2]. Characteristic clinical symptoms of OA include pain, restricted jaw movement and joint noise, particularly grating or grinding.

Treatment for OA of the Temporomandibular Joint (TMJ) consists of the elimination of possible causal factors, conservative treatment (occlusion splints), minimally invasive treatment [arthrocentesis, corticosteroids, or Hyaluronic Acid (HA) injections], arthroscopy and arthrotomy. For extensive degenerative changes, in which the disease does not respond to any treatment modality, joint replacement is indicated [3-5].

Platelet-rich plasma (PRP) has been used medicinally since the 1970s [6] and is obtained from autologous blood because the platelet concentration in PRP is at least 5-fold greater than that in fysiological blood. PRP is prepared by centrifuging autologous blood with anticoagulants, such as sodium citrate, which cause the blood to separate into 3 layers in the test tube: Platelet Poor Plasma (PPP), PRP and some red blood cells [6,7]. Blood platelets have no nuclei and are formed through megakaryocyte fragmentation of bone marrow. The physiological blood platelet count range is $200,000-400,000 / \mu \mathrm{L}$. Blood platelets contain the following granules [6]: alpha - contains fibrinogen, thrombospondin, and growth factors; GFs, delta - contains serotonin, ionized calcium, pyrophosphate, adenosine triphosphate, and adenosine diphosphate, and lambda - lysosomes containing lysosomal enzymes.

Growth Factor (GF) contained in alpha granules are particularly vital to healing and tissue regeneration and include Transforming Growth Factor beta-I (TGF- $\beta 1$ ) which controls proliferation, differentiation, and other functions in many cell types; Platelet-Derived Growth Factor Alpha (PDGFA) which is a potent mitogen for connective tissue cells exerting its function by interacting with related receptor tyrosine kinases; Platelet-Derived Growth Factor Beta (PDGFB), which promotes cellular proliferation and inhibits apoptosis; platelet-derived growth factor C - PDGFC that increases motility in mesenchymal cells, fibroblasts, smooth muscle cells, capillary endothelial cells, and neurons; Insulin-Like Growth Factor I (IGF-1), that mediates many of the growth-promoting effects of growth hormones; Fibroblast Growth Factor I (FGF1) which induces liver gene expression, angiogenesis, and fibroblast proliferation; Epidermal Growth Factor (EGF), that induces differentiation of specific cells and is a potent mitogenic factor for a variety of cultured cells of both ectodermal and mesodermal origin; Vascular Endothelial Growth Factor A (VEGFA), that is a mitogen found in vascular endothelial cells and induces angiogenesis; Vascular Endothelial Growth Factor B (VEGFB), which is a regulator of

*Corresponding author: Vladimír Machoň, Department of Oral Maxillofacia Surgery, General University Hospital, Prague, U Nemocnice 2, 120 00, Prague, Czech Republic, Tel: +420-721151540; E-mail: machonv@seznam.cz

Received September 06, 2013; Accepted October 17, 2013; Published October 24,2013

Citation: Machoň V, Řehořová M, Šedý J, Foltán R (2013) Platelet-Rich Plasma in Temporomandibular Joint Osteoarthritis Therapy: A 3-Month Follow-Up Pilot Study. J Arthritis 2: 112. doi: 10.4172/2167-7921.1000112

Copyright: (c) 2013 Machoň V W, et al. This is an open-access article distributed under the terms of the Creative Commons Attribution License, which permits unrestricted use, distribution, and reproduction in any medium, provided the original author and source are credited. 
blood vessel physiology with a role in endothelial targeting of lipids to peripheral tissues; and Vascular Endothelial Growth Factor C (VEGFC), that promotes angiogenesis and endothelial cell growth and affects blood vessel permeability. The presence of these GF peptides and their application in high concentrations to damaged tissue, a fracture, or inter-articularly is the principle behind PRP therapy [6-12].

PRP is used particularly in orthopedic support therapy for injured tendons and ligaments and in regeneration of OA-induced changes to cartilage and subchondral bone $[8,13,14]$. The objective of the present study is to clarify the effect of PRP in patients with OA of the TMJ during a 3-month prospective study.

\section{Materials and Methods}

\section{Patients}

A pilot sample cohort was comprised of 30 OA patients (Wilkes stage IV). The study subjects included 3 males and 27 females with an average age of $33.4 \pm 2.0$ years (range, 17-65 years). The study only included patients with a unilateral TMJ disability without systemic joint disease (rheumatoid arthritis, hormonal changes, etc.). All patients underwent the following 3- stage therapy without any symptom resolution.

\section{Design on the study}

All patients in this study underwent:

1. six months of conservative treatment (soft diet, analgesics, and occlusion splint)

2. minimally -invasive treatment ( 2 arthrocenteses with irrigation treatment using 100-120 mL of Ringer solution and HA instillation at the end of the lavage)

3. arthroscopic lavage (irrigation treatment using $800-1000 \mathrm{~mL}$ of Ringer solution).

Subsequently, patients who did not improve after arthroscopic lavage and had arthroscopic conditions characterized by inflammation of the synovial and retrodiscal tissues (tissue hyperemia and hyperplasia) were added to the study. During arthroscopy, the quality of the joint cartilage was assessed. The presence of chondromalacia was determined [15]: Stage I- softening of cartilage, reduced ability to withstand additional compressive and shearing forces; Stage IIblistering of the cartilage surface and formation of furrows and blisters; Stage III- fissuring and fibrillation of the articular surface and Stage IV- exposure of subchondral bone. Only patients with stage I and II chondromalacia were included in this study, whereas patients with disc dislocation, disc perforation, or stage III and IV chondromalacia were excluded.

The patients were randomly divided into 3 groups: the first 10 patients that fulfilled the study criteria comprised group A, the next 10 into group B, and the last 10 into group C. The patients in group A were administered PRP (2 applications with a 2- week interval), while the patients in group $\mathrm{B}$ were administered sodium hyaluronate into the joint ( 2 applications with a 2-week interval). The patients in group C (the control group) did not undergo any treatment, but merely continued to use an occlusion splint (a mandibular stabilization plate 3 mm thick, hard, full coverage dental arch) and analgesics (Paracetamol $500 \mathrm{mg}$, 3 times daily for 1 week, and thereafter in the case of further).

\section{Clinical assessment}

The patients subjectively assessed their pain intensity using the Visual Analog Scale (VAS; scale 0-10) and the Maximum Interincisal
Opening (MIO) was clinically measured in millimeters. This assessment was performed prior to and then at a follow-up examination 3 months after the administration of PRP or HA, or 3 months after the commencement of conservative therapy.

\section{Preparation of PRP}

PRP was prepared from a $10 \mathrm{~mL}$ autologous venous blood sample taken from the antecubital vein. The blood was drawn into test tubes containing sodium citrate and then centrifuged for $6 \mathrm{~min}$ at $1500 \mathrm{rpm}$. The blood was separated into 3 layers: a red bottom layer containing red blood cells; a pink middle layer containing PRP; and a yellow top layer containing PPP. The middle plasma layer (PRP) was then drawn from the test tubes using a micropipette.

\section{Intra-articular application}

The needle insertion point was locally disinfected and the lateral TMJ fossa rim and condylar head were located by palpation. The patient was then asked to open their mouth and a 19 gauge needle was inserted $10 \mathrm{~mm}$ in front of the tragus and $2 \mathrm{~mm}$ below the lateral canthotragal line. Then, 1-2 mL of anesthetic (4\% Articain with Epinephrine), was injected into the joint and then aspirated back into the syringe. In cases where aspiration was possible, the needle was clearly directed intraarticularly [5]. (The TMJs of Group A patients were injected with $1 \mathrm{~mL}$ of PRP; those in group B were administered $1 \mathrm{ml}$ of sodium hyaluronate. The injection sites were covered with a sterile bandage. The procedure was always performed with a prophylactic dose of antibiotics ( $2 \mathrm{~g}$ of Amoxicilin; in patients allergic to penicillin, Clindamycin $600 \mathrm{mg}$ was used 1 hour before the procedure).

\section{Post-operative care}

The same post-operative care regimen was applied to all patients after intra-articular application: for 3 days immediately following the injection, patients were instructed to open their mouths as little as possible; afterwards, they were instructed to open their mouths to the threshold of pain. All subjects in the study were informed to wear an occlusion splint 24 hours a day for the first 3 days, then regularly at night. During the 3 days immediately following injectuion, all patients regularly received analgesics (Paracetamol $500 \mathrm{mg}, 3$ times daily); afterwards they only received analgesics in the case of pain.

\section{Statistical analysis}

Data are reported as (i) absolute numbers, (ii) percentage of the whole sample or (iii) mean \pm S.E.M. Inter-group differences between particular groups as well as in-group differences inside particular groups were analyzed by non-paired or paired Student's T-test. Statistically significant differences was set for $\mathrm{p}<0.05$. All efforts have been made to eliminate potential sources of bias. The study has been designed and reported in accordance with the STROBE statement.

\section{Results}

\section{Range of opening}

The overall average interincisal mouth opening before treatment was $30.5 \pm 1.8 \mathrm{~mm}$ (range $16-39 \mathrm{~mm}$ ). At the 3-month follow-up, patients from both groups, $A$ and $B$, improved their mouth opening. The average interincisal mouth opening was $31.2 \pm 1.8 \mathrm{~mm}$. The mean difference in mouth opening prior to and 3 months after treatment were $1.6 \pm 1.0$ ( $5 \%$ of original value) in group A, $0.6 \pm 0.4 \mathrm{~mm}$ ( $2 \%$ of original value) in group $\mathrm{B}$, and none in control group $\mathrm{C}$. The difference in this parameter between groups $A$ and $B$ was not significant $(p=0.39)$. 


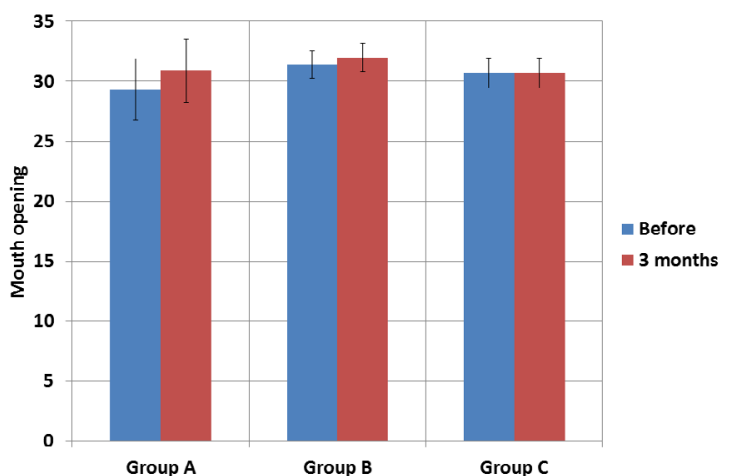

Figure 1: Interincisal mouth opening in patients from groups $A, B$ and $\mathrm{C}$ before treatment and during 3-month follow-up period after particular treatment (mean $\pm \mathrm{S}$. E. M). The differences between both groups were not statistically significant $(p>0.05)$.

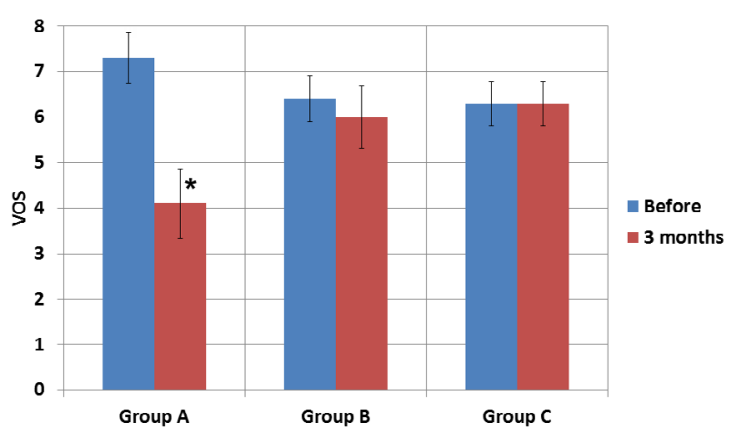

Figure 2: Pain intensity, reflected by VAS (range 0-10; mean \pm S. E. M) in patients from groups $A, B$ and $C$ before treatment and during 3-month follow-up period after treatment. Significant difference $(p>0.05)$ is marked with asterisk.

Moreover, there was no statistical difference $(\mathrm{p}>0.05)$ between groups $\mathrm{A}$ and $\mathrm{B}$ in interincisal mouth opening before as well as during 3-month follow-up period (Figure 1).

\section{Pain evaluation}

Three months after application, patients from both group A and $\mathrm{B}$ had decrease of pain intensity (Figure 2). Of the 10 patients who underwent PRP therapy, 7 (70\%) experienced a reduction in pain at their 3- month follow-up examination. On contrary, of the 10 patients who underwent sodium hyaluronate injection, only 2 (20\%) reported a reduction in pain at their 3- month follow-up examination. After treatment, pain relief was significantly more pronounced in group A-the difference between groups A and B in VAS after 3-month follow-up was significant $(\mathrm{p}=0.047)$. Subsequently, in-group differences are evaluated with paired Student's T-test. In group A, mean VAS decreased from 7.3 \pm 0.55 to $4.1 \pm 0.77$, corresponding to mean decrease of $3.2 \pm 0.81(44 \%$ of original value). This difference was significant ( $\mathrm{p}=0.005)$. In group $\mathrm{B}$, mean VAS decreased from $6.4 \pm 0.50$ to $6.0 \pm 0.68$, corresponding to mean decrease of $0.4 \pm 0.25$ ( $6 \%$ of original value). This difference was not significant $(\mathrm{p}=0.66)$ (Figure 2$)$.

\section{Discussion}

Minimally invasive modalities are typically utilized in the management of early stage osteoarthritis [16]. Minimally invasive treatment includes intra-articular administration of $\mathrm{HA}$ and corticosteroids, and arthrocentesis [3,4,16]. Bjornland et al. compared the effect of 2 injections of sodium hyaluronate (HA) with that of corticosteroids in 40 patients with TMJ OA. The HA patients had significantly better pain relief [17]. Moystad et al. found no significant difference with 2 intra-articular injections of HA and corticosteroids [18]. Similarly, Kopp found no significant difference between the efficacies of HA and corticosteroids [3].

Intra-articular administration of anti-inflammatory drugs into joints can improve lubrication. In contrast, arthrocentesis provides joint lavage, the irrigation of inflammatory mediators and loose particles of cartilage, thus loosening adhesions [5,16]. Nevertheless, many physicians prefer to use HA at the end of arthrocentesis for its positive effect on inflammatory degenerative disorders [4,19], but HA is more effective in the early stages of OA than in stages characterized by morphological changes of the articular surfaces $[5,19]$. Manfredini et al. compared several arthrocentesis injection options and obtained better results with HA compared to corticosteroids [4]. Noticeable improvement was achieved with repeated arthrocentesis combined with HA application [4,19]. Guarda-Nardini et al. reported improvement after only 2 arthrocenteses combined with HA instillation [19]. In the Guarda-Nardini study, the effect of the application of sodium hyaluronate was significantly lower-only $20 \%$, but it is important to understand that the study dealt only with patients unresponsive to conventional treatment methods (conservative treatment, 2 arthrocentesis therapies combined with HA administration and arthroscopic lavage, but always with no effects) [19]. In the present study, the patients obtained a higher therapeutic effect from intra-articular administration of PRP-70\% of patients reported reduced pain and improved mouth opening.

PRP therapy is based on the effects of GFs that promote changes in cell proliferation and regulate cellular metabolism. GFs have a vital role in modulating chondrogenic expression [20]. PRP promotes healing through regeneration of degenerative changes in cartilage, bone, and synovial tissue [7]. In oral surgery, PRP is used to support bone regeneration in sinus lifts, cleft surgery, and jaw reconstruction, promotes healing of oroantral communications, bone defects, and cysts, and prevents $f$ the development of dry socket [7,21]. PRP is widely used in orthopedic surgery, as it promotes healing of wounded tendons and ligaments (medial collateral ligament rupture of the knee, lateral collateral ligament rupture of the ankle and tendon defects) and regenerates OA damage to cartilage and subchondral bone $[8,13,14]$.

While articular cartilage has limited regenerative capacity due to its avascularity and low mitotic activity, some GFs especially TGF beta, basic fibroblast growth factor, and bone morphogenic protein show a positive effect on cartilage tissue regeneration [10,22,23]. The effect of PRP on inflammatory degenerative changes has been described in many studies. In a study of porcine knee arthritis, Lippross et al. reported that PRP reduced inflammatory mediator synthesis in the synovial membrane [24]. These results were confirmed by Liu et al. in a study of rabbit knee synovitis [25]. Sun et al. reported improved healing of osteochondral defects in rabbits, and Ying et al. reported a positive effect of TGF in OA of rabbit TMJs [11,26].

Several clinical trials have shown that PRP therapy is effective for pain reduction and subsequent improvement in joint function. Sánchez et al. treated 40 patients with OA of the hip utilizing intra-articular PRP and found that $57.5 \%$ of patients achieved pain reduction on a 6-month follow-up and 16 patients had excellent results [10]. Significant pain reduction was also reported by Filardo et al., who evaluated the use of PRP in 91 patients in a 12-month follow-up study of chronic 
degeneration of the knee [27]. Sampson et al. noted a reduction in pain and disease improvement in 14 patients with OA of the knee [28]. Similar results were reported by Napolitano et al. in 27 patients with OA of the knee and by Kon et al. in 150 patients with OA of the knee [20,29].

In the present study, PRP was used in patients with OA of the TMJ. Pain reduction and improvement in jaw mobility occurred in $70 \%$ of the participants. In this study, PRP was injected into the affected TMJs 2 times in succession with a 2-week interval. However, many orthopedic studies have reported a greater number of repeated applications, mostly 3 at an interval of 3-5 weeks [10,13,20,22,2729]. As in other studies, there were no complications related to the administration of PRP [13,20,27-29]. Contraindications for the use of PRP include platelet dysfunction syndrome, critical thrombocytopenia, hypofibrinogenemia, and hemodynamic instability [30].

\section{Conclusion}

Our results demonstrated the early efficacy of PRP in patients with $\mathrm{OA}$ of the TMJ as a possible alternative treatment. In patients who did not respond to standard treatment, PRP administration showed a significantly greater effect than sodium hyaluronate.

This was a small pilot study and the study patients were only subjected to intra-articular administration of PRP or sodium hyaluronate without simultaneous arthrocentesis. Future, large cohort studies are required to evaluate the effectiveness of the present methodology compared to other minimally invasive treatment modalities, the exact biological effects of PRP in OA, and to determine the best management algorithm for early TMJ OA.

\section{Grant support}

\section{GAUK UK 338411}

\section{References}

1. Emshoff R, Puffer P, Rudisch A, Gassner R (2000) Temporomandibular joint pain: relationship to internal derangement type, osteoarthrosis, and synovial fluid mediator level of tumor necrosis factor-alpha. Oral Surg Oral Med Oral Pathol Oral Radiol Endod 90: 442-449.

2. Stegenga B, de Bont LG, Boering G (1989) Osteoarthrosis as the cause of craniomandibular pain and dysfunction: a unifying concept. J Oral Maxillofac Surg 47: 249-256.

3. Kopp S, Carlsson GE, Haraldson T, Wenneberg B (1987) Long-term effect of intra-articular injections of sodium hyaluronate and corticosteroid on temporomandibular joint arthritis. J Oral Maxillofac Surg 45: 929-935.

4. Manfredini D, Rancitelli D, Ferronato G, Guarda-Nardini L (2012)Arthrocentesis with or without additional drugs in temporomandibular joint inflammatorydegenerative disease: comparison of six treatment protocols*. J Oral Rehabil 39: $245-251$

5. Nitzan DW, Dolwick MF, Martinez GA (1991) Temporomandibular join arthrocentesis: a simplified treatment for severe, limited mouth opening. J Oral Maxillofac Surg 49: 1163-1167.

6. Sánchez-González DJ, Méndez-Bolaina E, Trejo-Bahena NI (2012) Plateletrich plasma peptides: key for regeneration. Int J Pept 2012: 532519

7. Prakash S, Thakur A (2011) Platelet concentrates: past, present and future. J Maxillofac Oral Surg 10: 45-49.

8. Park YG, Han SB, Song SJ, Kim TJ, Ha CW (2012) Platelet-rich plasma therapy for knee joint problems: review of the literature, current practice and legal perspectives in Korea. Knee Surg Relat Res 24: 70-78.

9. Pujol JP, Chadjichristos C, Legendre F, Bauge C, Beauchef G, et al. (2008) Interleukin-1 and transforming growth factor-beta 1 as crucial factors in osteoarthritic cartilage metabolism. Connect Tissue Res 49: 293-297.
10. Sánchez M, Anitua E, Azofra J, Aguirre JJ, Andia I (2008) Intra-articular injection of an autologous preparation rich in growth factors for the treatment of knee OA: a retrospective cohort study. Clin Exp Rheumatol 26: 910-913.

11. Sun $Y$, Feng $Y$, Zhang $C Q$, Chen SB, Cheng XG (2010) The regenerative effect of platelet-rich plasma on healing in large osteochondral defects. Int Orthop 34: 589-597.

12. Tanimoto K, Suzuki A, Ohno S, Honda K, Tanaka N, et al. (2004) Effects of TGF-beta on hyaluronan anabolism in fibroblasts derived from the synovial membrane of the rabbit temporomandibular joint. J Dent Res 83: 40-44.

13. Li M, Zhang C, Ai Z, Yuan T, Feng Y, et al. (2011) [Therapeutic effectiveness of intra-knee-articular injection of platelet-rich plasma on knee articular cartilage degeneration]. Zhongguo Xiu Fu Chong Jian Wai Ke Za Zhi 25: 1192-1196.

14. Lyras D, Kazakos K, Verettas D, Polychronidis A, Simopoulos C, et al. (2010) Immunohistochemical study of angiogenesis after local administration of platelet-rich plasma in a patellar tendon defect. Int Orthop 34: 143-148.

15. Tarro AW TMJ Arthroscopy (1993) A Diagnostic and Surgical Atlas. J.B. Lippincott Comp., Philadelphia 198 pp.

16. Nitzan DW, Price A (2001) The use of arthrocentesis for the treatment of osteoarthritic temporomandibular joints. J Oral Maxillofac Surg 59: 1154-1159.

17. Bjørnland T, Gjaerum AA, Møystad A (2007) Osteoarthritis of the temporomandibular joint: an evaluation of the effects and complications of corticosteroid injection compared with injection with sodium hyaluronate. J Oral Rehabil 34: 583-589.

18. Møystad A, Mork-Knutsen BB, Bjørnland T (2008) Injection of sodium hyaluronate compared to a corticosteroid in the treatment of patients with temporomandibular joint osteoarthritis: a CT evaluation. Oral Surg Oral Med Oral Pathol Oral Radiol Endod 105: e53-60.

19. Guarda-Nardini L, Stifano M, Brombin C, Salmaso L, Manfredini D (2007) A one-year case series of arthrocentesis with hyaluronic acid injections for temporomandibular joint osteoarthritis. Oral Surg Oral Med Oral Pathol Oral Radiol Endod 103: e14-22.

20. Napolitano M, Matera S, Bossio M, Crescibene A, Costabile E, et al. (2012 Autologous platelet gel for tissue regeneration in degenerative disorders of the knee. Blood Transfus 10: 72-77.

21. Carlson NE, Roach RB Jr (2002) Platelet-rich plasma: clinical applications in dentistry. J Am Dent Assoc 133: 1383-1386.

22. Sánchez M, Guadilla J, Fiz N, Andia I (2012) Ultrasound-guided platelet-rich plasma injections for the treatment of osteoarthritis of the hip. Rheumatology (Oxford) 51: 144-150.

23. van Buul GM, Koevoet WL, Kops N, Bos PK, Verhaar JA, et al. (2011) Platelet-rich plasma releasate inhibits inflammatory processes in osteoarthritic chondrocytes. Am J Sports Med 39: 2362-2370.

24. Lippross S, Moeller B, Haas H, Tohidnezhad M, Steubesand N, et al. (2011) Intraarticular injection of platelet-rich plasma reduces inflammation in a pig model of rheumatoid arthritis of the knee joint. Arthritis Rheum 63: 3344-3353.

25. Liu J, Yuan T, Zhang C (2011) [Effect of platelet-rich plasma on synovitis of rabbit knee]. Zhongguo Xiu Fu Chong Jian Wai Ke Za Zhi 25: 285-290.

26. Ying B, Chen K, Hu J, Man C, Feng G, et al. (2013) Effect of different doses of transforming growth factor- $\hat{I}^{2} \hat{a}$, on cartilage and subchondral bone in osteoarthritic temporomandibular joints. Br J Oral Maxillofac Surg 51: 241-246.

27. Filardo G, Kon E, Buda R, Timoncini A, Di Martino A, et al. (2011) Platelet-rich plasma intra-articular knee injections for the treatment of degenerative cartilage lesions and osteoarthritis. Knee Surg Sports Traumatol Arthrosc 19: 528-535.

28. Sampson S, Reed M, Silvers H, Meng M, Mandelbaum B (2010) Injection of platelet-rich plasma in patients with primary and secondary knee osteoarthritis: a pilot study. Am J Phys Med Rehabil 89: 961-969.

29. Kon E, Mandelbaum B, Buda R, Filardo G, Delcogliano M, et al. (2011) Plateletrich plasma intra-articular injection versus hyaluronic acid viscosupplementation as treatments for cartilage pathology: from early degeneration to osteoarthritis. Arthroscopy 27: 1490-1501.

30. Floryan KM, Berghoff WJ (2004) Intraoperative use of autologous platelet-rich and platelet-poor plasma for orthopedic surgery patients. AORN J 80: 668-674 quiz 675-8. 